\title{
The Role of cGMP-Dependent Signaling Pathway in Synaptic Vesicle Cycle at the Frog Motor Nerve Terminals
}

\author{
Aleksej M. Petrov, ${ }^{1}$ Arthur R. Giniatullin, ${ }^{1}$ Guzel F. Sitdikova, ${ }^{2}$ and Andrej L. Zefirov ${ }^{1}$ \\ ${ }^{1}$ Department of Normal Physiology, Kazan State Medical University, 420012 Kazan, Russia, and ${ }^{2}$ Department of Human and Animals Physiology, Kazan \\ State University, 420008 Kazan, Russia
}

The role of cGMP-dependent pathways in synaptic vesicle recycling in motor nerve endings during prolonged high-frequency stimulation was studied at frog neuromuscular junctions using electrophysiological and fluorescent methods. An increase of intracellular cGMP concentration (8-Br-cGMP or 8-pCPT-cGMP) significantly reduced the cycle time for synaptic vesicles through the enhancement of vesicular traffic rate from the recycling pool to the readily releasable pool and acceleration of fast endocytosis. Pharmacological inhibition of soluble guanylate cyclase or protein kinase $\mathrm{G}$ slowed down the rate of recycling as well as endocytosis of synaptic vesicles. The results suggest that cGMP-PKG-dependent pathway serves a significant function in the control of vesicular cycle in frog motor terminals.

Key words: exocytosis; endocytosis; recycling of synaptic vesicles; cGMP; FM1-43; end-plate currents

\section{Introduction}

Presynaptic nerve terminals at chemical synapses contain many synaptic vesicles (SVs) which can be stored, transported and released in a highly coordinated manner. An increase in the level of intraterminal $\mathrm{Ca}^{2+}$ leads to fusion of vesicles with the presynaptic membrane at the active zones in the terminal resulting in the release of neurotransmitter into the synaptic cleft (Couteaux and Pécot-Dechavassine, 1970; Sudhof, 2004). After the fusion event, new SVs are formed by endocytosis. These vesicles enter the nerve terminal, where they are refilled with neurotransmitter and can be used again. The constant turnover of vesicles in the nerve terminal is a SV cycle (Sudhof, 2004; Kavalali, 2006). The rate of vesicular cycle has a special significance during prolonged highfrequency activity, when it may limit the magnitude of postsynaptic signals. According to the currently held view, the regulation of vesicle turnover rate may contribute to the mechanisms of synaptic plasticity (Micheva et al., 2003; Kavalali, 2006).

Analysis of the different steps of the cycle indicates an important role of phosphorylation/dephosphorylation processes in its regulation (Cousin and Robinson, 2001; Wenk and Camilli, 2004). For example, the activation of protein kinases $A$ or $C$ at hippocampal synapses increased the probability of exocytosis of single docked vesicles (Snyder et al., 2006). During long lasting high-frequency activity at Drosophila neuromuscular junctions, the activation of protein kinase A mediated the movement of vesicles to sites of exocytosis (Kuromi and Kidokoro, 2005). However, the inhibition of protein kinases with staurosporine

Received June 26, 2008; revised 0ct. 10, 2008; accepted 0ct. 15, 2008.

This work was supported by Russian Foundation for Basic Research Grant No. 08-04-00203 and Russian Ministry of Education and Science Grant NSh-3368.2008.4. We thank Vadim Bolshakov and Clark Slater for helpful comments on this manuscript.

Correspondence should be addressed to Aleksej M. Petrov, Department of Normal Physiology, Kazan State Medical University, 49 Butlerova Street, 420012 Kazan, Russia. E-mail: fysio@rambler.ru.

DOI:10.1523/JNEUROSCI.2947-08.2008

Copyright $\odot 2008$ Society for Neuroscience $\quad 0270-6474 / 08 / 2813216-07 \$ 15.00 / 0$ reduced the SVs mobilization and amplified the depression of postsynaptic responses at neuromuscular and hippocampal synapses (Kraszewski et al., 1996; Becherer et al., 2001). Okadaic acid, a protein phosphatase inhibitor, increased the mobility of SVs, disrupted the vesicle clusters and, as a result, decreased the transmitter release from motor nerve terminals or spinal cord neurons (Betz and Henkel, 1994; Dai and Peng, 1996). It has been shown previously that a NO-dependent increase of cGMP level accelerated SVs recycling in the hippocampus (Micheva et al., 2003). Most of the observed effects of cGMP are mediated by protein kinase G (PKG) activation (Wang et al., 2005). Some proteins participating in the processes of exo/endocytosis and SVs trafficking can be phosphorylated by PKG (Xue et al., 2004). In hippocampal neurons PKG mediates the presynaptic component of long-term depression (Feil et al., 2003) and long-term potentiation (Kleppisch et al., 2003, Stanton et al., 2003). Despite these previous efforts, the role of cGMP-system in exocytosis and endocytosis processes is still largely unknown.

In the present study, electrophysiological and fluorescent methods have been used to investigate the role of cGMP and PKG in the processes of transmitter release, exocytosis, endocytosis and recycling at the frog motor nerve terminals during prolonged high-frequency activation. We addressed the following questions: How does the increase of cGMP-system activity affect acetylcholine release, exo/endocytosis and recycling of SVs during high-frequency activation? Is this system tonically active in nerve terminals? Are the effects of cGMP mediated by PKG?

\section{Materials and Methods}

Preparation and solution. Experiments were performed on frog (Rana ridibunda) sartorius or cutaneous muscle preparation in vitro at room temperature. Muscles stretched and fixed in the glass sylgard lined chamber containing frog Ringer's solution (mM): $\mathrm{NaCl} 113, \mathrm{KCl} 2.5, \mathrm{CaCl}_{2} 1.8$, $\mathrm{NaHCO}_{3} 2.4$ (pH 7.2-7.4, 22 ${ }^{\circ} \mathrm{C}$ ). Solution of $100 \mu \mathrm{M} \mathrm{8-bromo-cyclic}$ GMP and $50 \mu \mathrm{M}$ 8-para-chlorophenylthio-cyclic GMP were prepared immediately before use to avoid light-induced inactivation. $1 H-(1,2,4)$ 
oxidazole (4,3-a) quinoxalin-1-one (ODQ) was prepared as stock solution in DMSO and added to normal Ringer's solution immediately before use. After dilution of ODQ $(1 \mu \mathrm{M})$ DMSO concentration was no more that $0.001 \%$. Stock solution of Rp-8-bromo- $\beta$-phenyl- $1, N^{2}$ etheno-guanosine- $3^{\prime}, 5^{\prime}$-cyclic monophosphorothioate (Rp-8-Br-PETcGMP) was prepared in distilled water, aliquoted and stored at $-20^{\circ} \mathrm{C}$. $\mathrm{Rp}-8$-Br-PET-cGMP was used at $0.5 \mu \mathrm{M}$, in this concentration it mainly blocks the activity of protein kinase G1 (Schwede et al., 2000). $\mathrm{N}$-[3(triethylammonium) propyl]-4-(4-dibutilaminostyryl pyridinium, dibromide (FM1-43) and ADVASEP-7 were dissolved in water and stored at $4^{\circ} \mathrm{C}$. All drugs were dissolved to a final concentration in the bathing solution and applied in the chamber with neuro-muscular preparation (5 $\mathrm{ml}$ ) via a superfusion system with the rate of $1 \mathrm{ml} \mathrm{min}^{-1}$. All reagents were from Sigma except FM1-43 and ADVASEP-7, which were purchased from Biotium.

Animals were anesthetized with ether before being stunned and were pithed in accordance with the European Communities Council Directive (November 24, 1986; 86/609/EEC).

Electrophysiology. Recording of postsynaptic end-plate currents (EPCs) (see Fig. 1A) was performed using the standard two-electrode voltage-clamp technique with intracellular electrodes (3-5 M $\Omega$; filled with $2.5 \mathrm{M} \mathrm{KCl}$ ). To prevent muscle contraction the muscle fibers were cut transversely. The holding potential for cut preparations was kept at $-40 \mathrm{mV}$ (Glavinović, 1979). The motor nerve was stimulated with a suction electrode by a single $(0.2 \mathrm{~Hz})$ or high-frequency nerve stimulation. Evoked EPCs were digitized at $50 \mathrm{kHz}$ and analyzed off-line to calculate mean values of EPCs amplitudes.

Fluorescence microscopy. Fluorescent styryl dye FM1-43 (6 $\mu \mathrm{M})$ was used to estimate the rate of exocytosis and endocytosis of SVs. FM1-43 reversibly binds to presynaptic membrane and becomes trapped within recycled SVs during endocytosis-FM1-43 loading. The fluorescent labeling pattern in living frog neuromuscular preparation consists of a series of discrete spots 1-2 $\mu \mathrm{M}$ in diameter (see Fig. $3 C$ ), distributed along the length of motor nerve terminal (Betz and Bewick, 1993, Zefirov et al., 2003).

Fluorescence images were acquired by Olympus BX51W1 fluorescence microscope equipped with a $60 \times, 0.9$ numerical aperture, waterimmersion objective from LUMPLFL series (Olympus) and confocal attachment Disk Speed Unit (Olympus). Excitation light came from a 100 W Hg lamp, through 5-10\% neutral density transmission filters, excitation filter $(480 / 30 \mathrm{~nm})$, dichroic mirror $(505 \mathrm{~nm})$ and emission filter (535/40 nm). Multiple $z$-axis optical sections were taken using a focus stepper (ECO-MOT). Images were captured with CCD video camera F-VIEW-2 (0.5-2 s exposure) connected to a computer running for Windows imaging software. Image analysis was performed using programs CellP and Image Pro. For each preparation, four or five bright nerve terminals of different synapses were selected by eye for quantification of fluorescence intensity.

To determine the dynamics of endocytosis during or after highfrequency stimulation ( $20 \mathrm{~Hz}$ for $3 \mathrm{~min}$ ) next protocol was used. Motor nerve was stimulated at $20 \mathrm{~Hz}$ for a certain period of time (1, 2, $3 \mathrm{~min}$ ) and FM1-43 was added during last minute of stimulus trains (see Fig. $2 A)$. Such protocol was used to exclude the dye unloading during the following stimulation, because recycling time of SVs was estimated as $\sim 1$ min (Betz and Bewick, 1993; Zefirov et al., 2008). For example, to test endocytosis during first min of stimulation motor nerve was stimulated for $1 \mathrm{~min}$ and FM1-43 was added for $1 \mathrm{~min}$. To test endocytosis during second min of stimulation motor nerve was stimulated for $2 \mathrm{~min}$ and FM1-43 was added for second min and so on. After 1 min of FM1-43 application, the dye was washout from surface membrane by Ringer's solution containing ADVASEP-7 $(3 \mu \mathrm{M})$. This reagent accelerates dissociation of FM1-43 from the surface membranes and decreases nonspecific fluorescence. To estimate the dynamic of endocytosis after $3 \mathrm{~min}$ of $20 \mathrm{~Hz}$ stimulation, FM1-43 was added to the bath solution for $1 \mathrm{~min}$ with different delay time after the end of tetanus (see Fig. 2A). The fluorescence of terminal was calculated in arbitrary units (a.u.) as average brightness of all pixels of the part (selected spots) of terminal after subtraction of background fluorescence (Zefirov et al., 2006). The back- ground fluorescence value defined as an average brightness at the segment of terminal without spot (staining vesicle cluster).

To study the rate of destaining (see Fig. 3), terminals were loaded with FM1-43 (3 $\mu \mathrm{M})$ during $20 \mathrm{~Hz}$ nerve stimulation for $3 \mathrm{~min}$. The dye was applied for $3 \mathrm{~min}$ of $20 \mathrm{~Hz}$ stimulation and for $10 \mathrm{~min}$ after the stimulus train. Afterward the dye was washed out for $30 \mathrm{~min}$ and images were acquired. Average brightness levels of fixed fluorescent spots were measured.

Measurements are expressed as mean $\pm \operatorname{SEM}(n=$ number of experiments), with statistical significance assessed by Student's paired $t$ test. A $p$ value of $<0.05$ was accepted as indicative of statistically significant differences between groups.

\section{Results}

Modifying the activity of the cGMP-pathway does not affect transmitter release in response to low-frequency stimulation In control medium, the average amplitude, rise and decay time of EPCs evoked by low-frequency motor nerve stimulation $(0.2 \mathrm{~Hz})$ were $180 \pm 15 \mathrm{nA}, 0.31 \pm 0.03 \mathrm{~ms}, 1.2 \pm 0.04 \mathrm{~ms}$, respectively $(n=6)$. With the same stimulation protocol, previous exposure (40 min) to the membrane-permeable analogs of cGMP $(100 \mu \mathrm{M}$ 8-Br-cGMP, $n=6$ or $50 \mu \mathrm{M}$ 8-pCPT-cGMP, $n=6$ ) or guanylate cyclase and to PKG inhibitors ( $1 \mu \mathrm{M}$ ODQ, $n=7$ or $0.5 \mu \mathrm{M}$ Rp-8Br-PET-cGMP, $n=8$, respectively), had no effect on the amplitude $(176 \pm 12 \mathrm{nA} 8$-Br-cGMP, $174 \pm 14 \mathrm{nA} 8$-pCPTcGMP; $184 \pm 13$ nA ODQ; $183 \pm 12$ nA Rp-8Br-PET-cGMP), rise time $(0.29 \pm 0.03 \mathrm{~ms} 8$ - $\mathrm{Br}-\mathrm{cGMP}, 0.30 \pm 0.02 \mathrm{~ms} 8$-pCPTcGMP; $0.32 \pm 0.03 \mathrm{~ms}$ ODQ; $0.31 \pm 0.02 \mathrm{~ms}$ Rp-8Br-PETcGMP) or decay time $(1.22 \pm 0.03 \mathrm{~ms} 8$-Br-cGMP, $1.19 \pm 0.04$ ms 8-pCPT-cGMP; $1.21 \pm 0.05$ ms ODQ; $1.23 \pm 0.03$ ms Rp$8 \mathrm{Br}-\mathrm{PET}-\mathrm{cGMP}$ ) of EPCs.

\section{Membrane-permeable analogs of cGMP slow down depression of transmitter release at the beginning of prolonged high-frequency stimulation}

High-frequency stimulation $(20 \mathrm{~Hz}, 3 \mathrm{~min})$ under control conditions resulted in changes in the EPCs amplitude. We first observed a short period of facilitation, which was followed by the gradual reduction of the EPCs amplitude (Fig. $1 A-C$ ). By the 15 th second of stimulation, EPCs amplitude was reduced to $83 \pm$ $1.5 \%$, and by 30 th second, to $72 \pm 3 \%$ of the initial value. After 3 min stimulation, the EPCs amplitude was reduced to $16 \pm 2 \%$ $(n=6)$. It is well established, that the magnitude of depression of the EPCs amplitudes during presynaptic stimulation is determined mainly by the number of vesicles released from the nerve terminal in response to a single pulse (quantum content) and to a lesser extent on the size of transmitter quantum and the sensitivity of postsynaptic membrane to acetylcholine (Betz and Bewick, 1993; Rizzoli and Betz, 2005).

In the presence of cGMP analogs, the changes of EPCs amplitudes during high-frequency stimulation differed from those in the control medium. In the presence of 8 -Br-cGMP $(n=5)$ or 8 -pCPT-cGMP $(n=5)$, the EPCs amplitude was hardly reduced by $15 \mathrm{~s}$ of stimulation at $20 \mathrm{~Hz}$, and after $20 \mathrm{~s}$ it was only decreased to $91 \pm 1 \%(p<0.05)$ and $95 \pm 1 \%(p<0.05)$ respectively (Fig. $1 C)$. However, at longer stimulation times the decrease of the EPCs amplitude was more pronounced in cGMP analog-treated preparations than in control experiments. Thus at the end of a 3 min stimulation episode, the EPCs amplitude was reduced to $9 \pm$ $2 \%(p<0.05)$ of the initial value (Fig. $1 B)$

To compare the total number of quanta released by stimulation at $20 \mathrm{~Hz}$ for $3 \mathrm{~min}$ from nerve terminals in control medium and after exposure to cGMP analogs, cumulative curves of EPCs amplitudes during high-frequency stimulation were plotted. 
These showed that the application of cGMP analogs, leading to increases in intracellular cGMP concentration, did not change the amplitude of the summed EPCs relative to the control values (Fig. 1D).

\section{Inhibition of soluble guanylate cyclase and PKG induces depression of transmitter release during high- frequency stimulation}

The effects of ODQ on the time course of the EPCs amplitude during highfrequency stimulation differed from the action of cGMP analogs (Fig. $1 B$ ). At the beginning of stimulation, the EPCs amplitude decreased immediately and by the 15 th second of stimulation reduced to $63 \pm 1 \%(p<0.05)$ and by the 30th second, to $48 \pm 1 \%(p<0.05)$ of the initial level (Fig. 1C). At the end of the stimulus train, the EPCs amplitude was reduced to $9 \pm 1 \%(n=7, p<0.05)$. The summed amplitude of EPCs after ODQ application was smaller than in control experiments $(62 \pm 5 \%, p<0.05)$, indicating a decrease of total acetylcholine release from motor nerve terminal during high-frequency stimulation (Fig. 1D).

Similar changes were observed after inhibition of PKG (Fig. $1 A-D$ ). At the beginning of the stimulation episode, a significant decrease of the EPCs amplitude was observed (Fig. 1C). After $15 \mathrm{~s}$ stimulation, the EPCs amplitude decreased to $63 \pm 1 \%$ and by the end of 3 min stimulation, to $10 \pm 1.5 \%$ of the initial value. The summed amplitude of the EPCs after the treatment with Rp-8Br-PET-cGMP treatment was lower than under control conditions $(64 \pm 4 \%, n=8, p<0.05)$ (Fig. $1 D$ ).

\section{Treatment with membrane-permeable cGMP analogs} increases FM1-43 loading during high-frequency stimulation To determine the time course of FM1-43 loading, the dye was applied for $1 \mathrm{~min}$ during high-frequency stimulation $(20 \mathrm{~Hz}$ for 3 min) or after stimulation (Fig. 2A) (see Materials and Methods). In the control external solution, the intensities of nerve terminal fluorescence when exposed to FM1-43 during the first, second and third minutes of stimulation were $70 \pm 4(n=6), 74 \pm 5(n=$ 4), $77 \pm 5(n=5)$ a.u., respectively. The maximum intensity of fluorescence was observed after the end of stimulation ( 3 min 20 $\mathrm{Hz}$ ), in the fourth minute of the loading protocol (91 \pm 4 a.u., $n=$ 5). Afterward the fluorescence slowly decreased. By 3 min after the end of stimulation the intensity of fluorescence was $50 \%$ of the maximum and returned to the background value in 11-12 min (Fig. 2 B).

To reveal the total dye loading, loading during or after stimulation FM1-43 was added for $18 \mathrm{~min}$ ( 3 min during and 15 after stimulation), during $3 \mathrm{~min}$ or for $15 \mathrm{~min}$ after the end of stimulation (Fig. 2C). When FM1-43 was presented during and after stimulation the staining was brightest (655 \pm 45 a.u., $n=5)$. When dye was added during or only after the end of the stimulus train, the staining was $30 \pm 5 \%(p<0.05, n=5)$ or $70 \pm 8 \%$ $(p<0.05, n=5)$, respectively. Thus, under control conditions, the ratio of fluorescence intensity during and after stimulation was 30:70. (Fig. 2D).
Data in Figure $2 B$ does not take into account with findings of the Richards with colleagues (2000) that FM1-43 could gain access to large cisternae for long periods of time after stimulation stopped, but the dye could not wash back out. In our conditions (at all pharmacological influences) the brightness of nerve terminals loaded for $15 \mathrm{~min}$ after the end of stimulation close corresponded to the summed fluorescence of nerve terminals staining during per minute FM1-43 applications in poststimulation period. Therefore, twice labeling of the same vesicles by FM1-43 (when it was added with different delay time after stimulation) did not make a significant contribution in our estimation.

In the presence of cGMP analogs, the maximum level of fluorescence intensity reached during stimulation (Fig. $2 B$ ) and was higher than in the control medium (without drug application). After $3 \mathrm{~min}$ of stimulation the fluorescence intensity drastically reduced and by $4-5 \mathrm{~min}$ poststimulation the fluorescent labeling of nerve terminals was no longer visible. The analogs of cGMP decreased the total dye loading of nerve terminals to $70 \pm 6 \%$ (8-Br-cGMP, $p<0.05, n=5)$ and $68 \pm 5 \%$ (8-pCPT-cGMP, $p<$ $0.05, n=5$ ) of control. The ratio of fluorescence intensities at the time of stimulation and after was also changed. As shown in Figure $2 D$, this ratio was $\sim 60: 40$.

\section{Inhibition of guanylate cyclase and PKG reduces FM1-43 loading during prolonged high-frequency activation} Application of ODQ or Rp-8-Br-PET-cGMPS significantly decreased FM1-43 loading during high-frequency stimulation and prolonged the period of dye capture after stimulation of motor nerve. As shown in Figure $2 B$, there was no peak of fluorescence 

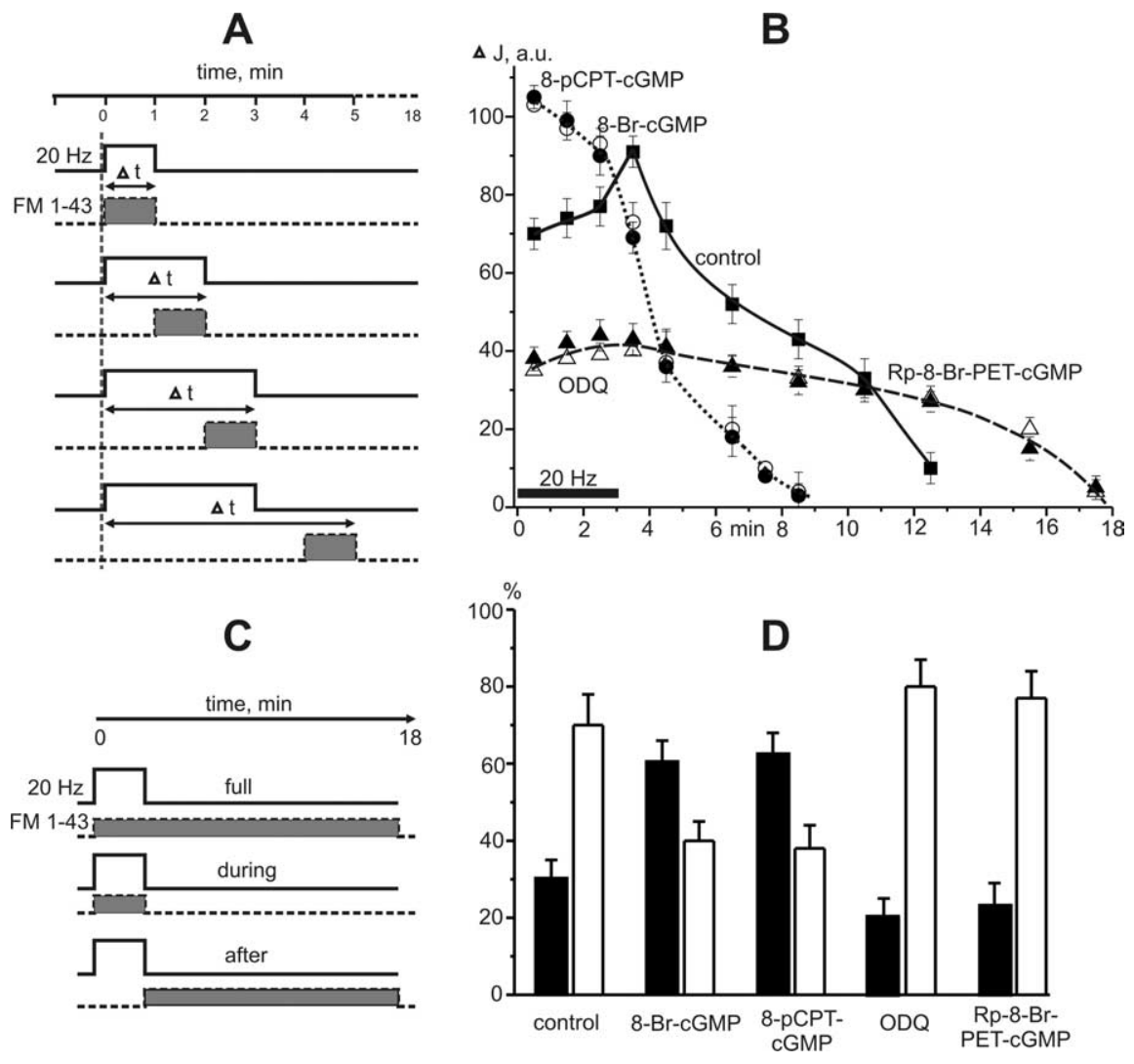

Figure 2. FM1-43 loading during and after high-frequency stimulation. $A$, A schematic diagram of the staining protocol. Light columns, with solid line, indicate the period of $20 \mathrm{~Hz}$ stimulation. Gray columns, with dotted line, show the time of FM1-43 presence for 1 min during $(\Delta t=1,2,3)$ and after the end of 3 min stimulation ( $\Delta t=4-18)$. FM1-43 was applied on the neuromuscular preparation for $1 \mathrm{~min}$ and then was quickly washed out with Ringer's solution containing ADVASEP-7 (3 $\mu \mathrm{m})$. $\boldsymbol{B}$, Time course of FM1-43 loading in motor nerve terminal. The intensity of nerve terminal fluorescence during and after highfrequency stimulation (per minute dye application). The first three points show the dye uptake during stimulation $(20 \mathrm{~Hz}, 3 \mathrm{~min})$ (horizontal line). $y$-axis, Fluorescence intensity ( $\Delta$ / in a.u.). C, A schematic diagram of the staining protocol. Light columns, with solid line, indicate the period of stimulation ( $3 \mathrm{~min} 20 \mathrm{~Hz}$ ). Gray columns, with dotted line, show the time of FM1-43 application. The total FM1-43 loading: dye was presented during (for $3 \mathrm{~min}$ ) and after (for $15 \mathrm{~min}$ ) tetanus. Loading during stimulation: dye was presented only during 3 min tetanus. Loading after stimulation: dye was added to chamber after the end of stimulus train for $15 \mathrm{~min}$. D, The comparison of dye amount (as a percentage) captured during and after high-frequency 3 min stimulation. The solid bars represent loading during stimulation while the open bars represent loading after stimulation. The total uptake of dye (when FM1-43 was presented during and after stimulation, see $\mathbf{C}$ ) was accepted as $100 \%$. When FM1-43 was presented only during or after stimulation, the nerve terminal staining was less than the brightness of terminals with total dye loading.

and the staining of nerve terminals did not change during the whole protocol (see Materials and Methods). The total dye loading after inhibition of guanylate cyclase or PKG was $74 \pm 4 \%$ ( $p<0.05, n=5$ ) of control. The uptake of dye during stimulation was only one third of that in control medium and most of the dye uptake occurred after simulation. The ratio of fluorescence intensities was $\sim 20: 80$ (Fig. $2 D$ ).

\section{Analogs of cGMP slow down the second phase of FM1-43 destaining from nerve terminal during prolonged high- frequency activity}

Under control conditions the activity-dependent nerve terminal destaining consisted of two components. The first component was characterized by a high rate of dye loss $(85 \pm 2 \%$ after $15 \mathrm{~s}$ of stimulation) (Fig. $3 B$ ). During the second phase, nerve terminal fluorescence reduced more slowly (Fig. $3 A$ ). At the end of the third and fifth minutes of stimulation, the intensity of fluorescent spots was reduced to $42 \pm 2 \%$ and $33 \pm 2 \%(n=7)$ of the initial value, respectively. After $15 \mathrm{~min}$ (900 s) period of stimulation, the labeling of vesicles was hardly detectable (Fig. 3C).
cGMP analogs did not change the first phase of nerve terminal destaining (Fig. $3 B$ ) but markedly slowed the rate of dye loss during the second phase (Fig. $3 A$ ). After incubation of nerve terminals in $8-\mathrm{Br}$ cGMP the fluorescence was reduced to $50 \pm 2$ and $41 \pm 2 \%(n=8)$ of the initial value by 3 and $5 \mathrm{~min}$ of stimulation, respectively. Application of 8-pCPT-cGMP reduced the nerve terminal fluorescence at 3 and 5 min of stimulation to $53 \pm 2$ and $47 \pm 3 \%$ of the initial values, respectively. In these terminals, dim fluorescent spots could still be detected even after $15 \mathrm{~min}$ simulation of motor nerve (Fig. 3C).

Inhibition of guanylate cyclase and PKG markedly slows the destaining of FM143 during prolonged stimulation

The inhibition of soluble guanylate cyclase and PKG markedly reduced the rate of dye loss, particularly at the beginning (first 20-30 s) of the high-frequency stimulation (Fig. $3 A, B$ ). After $15 \mathrm{~s}$ of stimulation the nerve terminal fluorescence was reduced to only $95 \pm 1 \%$ in the presence of ODQ $(p<0.05, n=8)$ and $97 \pm 1 \%$ in the presence of Rp-8-Br-PET-cGMP ( $p<$ $0.05, n=8)$. Subsequently, the rate of nerve terminals destaining was the same as in control experiments. After 3 and $5 \mathrm{~min}$ of stimulation, the intensity of fluorescence reduced to $65 \pm 1$ and $59 \pm 2 \%$ in the presence of ODQ, while in the presence of Rp-8-Br-PET-cGMP, to $65 \pm 1 \%$ and $56 \pm 2 \%$ of the initial values, respectively. After 15 min of tetanic stimulation, the fluorescence of the nerve terminal was still $\sim 30$ $35 \%$ of the initial value (Fig. $3 C$ ).

\section{Rise of intracellular cGMP reduces} whereas inhibition of guanylate cyclase and $P K G$ increases the $S V$ recycle time

\section{during high-frequency activity}

To estimate the recycle time of SVs during high-frequency activation we used technical approach previously described by Betz and colleagues (1993). In the context of their model, we compared the curves of dye loss and summed EPCs. For this purpose, the curve of dye loss was scaled and superposed with the curve of cumulative EPCs amplitude in their initial parts. This showed that the rate of dye loss eventually fell below the rate of quantal release. This deviation has been interpreted as being caused by recycling of unlabeled vesicles back into terminal, diluting the labeled pool and competing with labeled vesicles for release (Betz and Bewick, 1993; Reid et al., 2003). Exocytosis of such vesicles does not reduce the fluorescence intensity of the nerve terminal and thus slows down the rate of dye loss relative to that of transmitter release. The time when this deviation first becomes apparent has been termed the recycle time of vesicle $\left(t_{\mathrm{r}}\right)$ (Betz and Bewick, 1993). In our control experiments we found that the recycle time is $\sim 60 \mathrm{~s}$ (Fig. $4 \mathrm{~A}$ ), which is in a good agreement with the previously obtained estimates (Betz and Bewick, 1993; Reid et al., 2003; Zefirov et al., 2008). The recycle time of SVs was re- 
duced to $\sim 30$ s by exposure to 8 -Br-cGMP or 8-pCPT-cGMP (Fig. $4 B, C$ ). After the treatment with ODQ or Rp-8-Br-PETcGMP there was no deviation between the curves during $3 \mathrm{~min}$ of high-frequency stimulation, i.e., the recycle time was $>180$ s (Fig. $4 D, E)$.

At the neuromuscular junction of a mouse expressing synaptopHluorin it was shown that some synaptic vesicle might not contain acetylcholine and fused during intense stimulation (Tabares et al., 2007). In this case, if many fused synaptic vesicle or other organelles contain only dye and are acetylcholine-free then fluorescence will exceeded summed EPCs after superposed the curves in their initial parts, as was shown in this study. However, at frog motor nerve terminals participation of empty synaptic vesicles in neurotransmission during intense stimulation is not shown (Betz and Bewick, 1993; Richards et al., 2003).

\section{Discussion}

Here, we interpret our results in terms of current models regarding the vesicle cycle. Synaptic vesicles in frog motor nerve terminal can be divided functionally into at least three different pools, which sequentially participate in transmitter release. Readily releasable pool (RRP) is a small fraction $(2-4 \%)$ of all intraterminal vesicles, which are docked (and primed) to the presynaptic active zones and immediately available for release in response to stimulation (Becherer and Rettig, 2006). The second, mobilization pool contains $\sim 10-$ $20 \%$ of all vesicles (Elmqvist et al., 1964). Moderate physiological frequencies of stimulation cause continuous redistribution of vesicles between these two pools. Therefore, it was suggested that they could be treated as a single recycling pool (Rizzoli and Betz, 2005). Vesicles of this pool in frog nerve terminal recycle through a fast clathrin-dependent endocytotic pathway directly from the plasma membrane (Richards et al., 2000, 2003). High-frequency stimulation induces the depletion of the recycling pool and involvement of reserve pool vesicles (the third vesicular pool) in neurotransmission. The recovery of the reserve pool occurs by slow endocytosis including the formation of deep membrane infolding from which SVs are formed by clathrin-mediated endocytosis. Therefore the vesicles of the recycling pool are generally retrieved through fast endocytosis, by the so-called short pathway, whereas the release from the reserve pool is followed by slow endocytosis, by the so-called long pathway (Kuromi and Kidokoro, 2005; Rizzoli and Betz, 2005).

\section{Changes of cGMP-system activity do not modify transmitter} release during single-pulse stimulation

Our experiments indicate that neither inhibition of soluble guanylate cyclase or PKG, nor the increase of cGMP concentration,
A

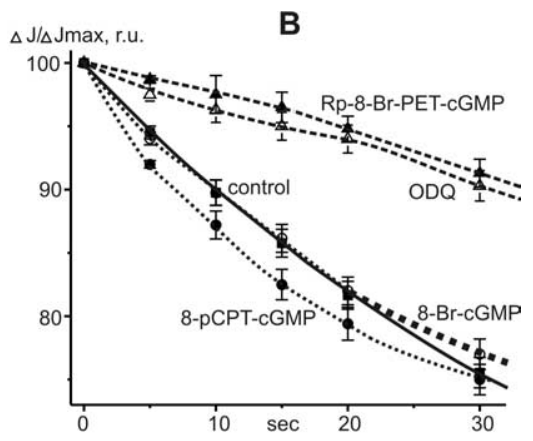

Figure 3. FM1-43 destaining kinetics in motor nerve terminals. $\boldsymbol{A}$, The decay of nerve terminal fluorescence during $20 \mathrm{~Hz}$ stimulation. $\boldsymbol{B}$, The curves of dye destaining during first 30 s of stimulation. $y$-axis, The relative fluorescence intensity $\left(\Delta / \Delta J_{\text {max }}\right)$, (00\% represents the intensity before tetanus. $\boldsymbol{X}$-axis, Time in minutes. $\boldsymbol{C}$, Change in fluorescent images of nerve terminals during tetanus in control and after treatment by 8-pCPT-cGMP or Rp-8-Br-PET-cGMP (0, 30, 90, 900 s of stimulation). Scale bars,
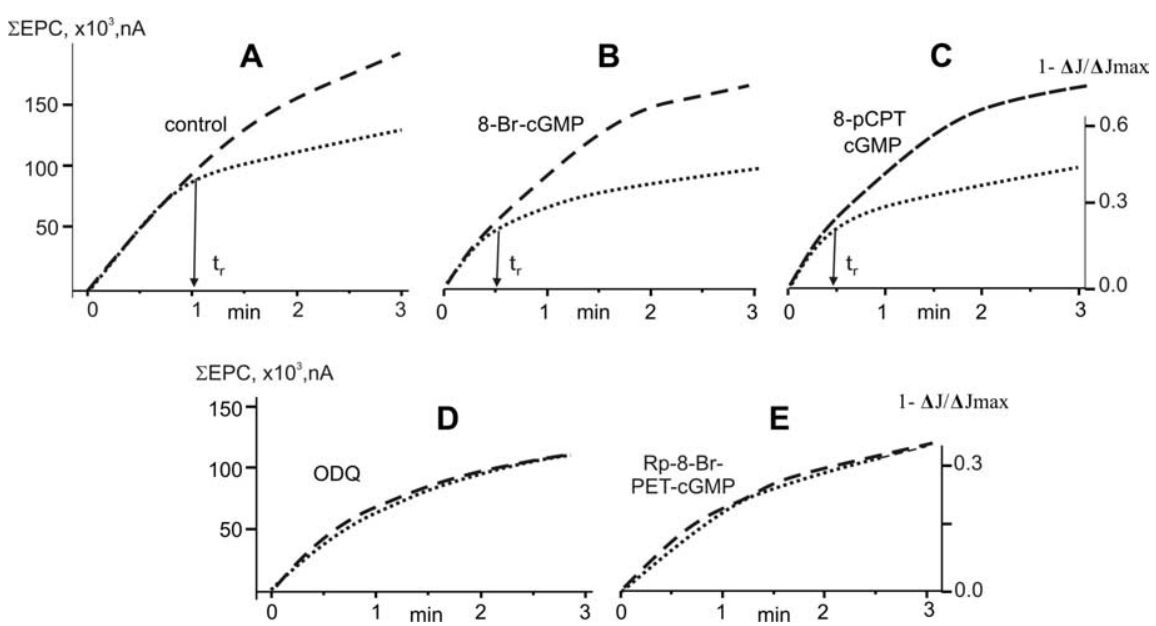

Figure 4. Calculation of the recycling time of synaptic vesicles. Superposition of the cumulative EPCs amplitude curve and dye loss curve during $20 \mathrm{~Hz}$ stimulation ( $3 \mathrm{~min}$ ). The dashed line, The cumulative EPCs amplitude curve (in nA) from Fig. 1; dotted line, dye loss curve from Figure 3 , which was scaled and superposed with the cumulative EPCs amplitude curve in their initial parts. The average recycle time $\left(t_{r}\right)$ is the point where the deviation between two curves becomes apparent. $\boldsymbol{A}$, Control; $\boldsymbol{B}, 8-\mathrm{Br}$-cGMP; $\boldsymbol{C}$ 8-pCPT-cGMP; $\boldsymbol{D}, 0 D Q ; \boldsymbol{F}, \mathrm{Rp}$-8-Br-PET-cGMP. $y$-axes, Summed EPCs amplitude in $\mathrm{nA}$ ( $\Sigma$ EPC, right axis), and the decrease of relative fluorescence intensity $\left(1-\Delta / \Delta J_{\text {max }}\right.$ left axis). $x$-axis, Time of stimulation in minutes.

affects transmitter release during single-pulse stimulation. Similar results were obtained in the study of Yakovlev et al. (2004), where cGMP analogs $\left(\mathrm{Bt}_{2}\right.$-cGMP, $\left.8 \mathrm{Br}-\mathrm{cGMP}\right)$ had no effects on transmitter release from frog nerve terminals. Our findings contrast, however, from a previous study, which reported a decrease of end-plate potential amplitude by 8 -Br-cGMP and an increase of transmitter release by an inhibitor of soluble guanylate cyclase, LY-83583 (Thomas and Robitaille, 2001). It is important to note that 8-pCPT-cGMP is 20 times more lipophilic than 8-Br-cGMP and is more resistant to hydrolysis (Schwede et al., 2000). However, ODQ is a more specific inhibitor of soluble guanylate cyclase ( $\mathrm{IC}_{50} 20 \mathrm{nM}$ ) than LY-83583, which could also have nonspecific effects (Lev-Ram et al., 1997). Importantly, PKG has no direct action on the exocytosis machinery (Snyder et al., 2006) 
and activation of the cGMP-dependent pathway does not affect the intracellular $\mathrm{Ca}^{2+}$ level (Thomas and Robitaille, 2001).

Activation of the cGMP-system increases fast endocytosis and recycling of SVs through the short pathway during prolonged high-frequency stimulation

Experiments with per minute FM1-43 loading revealed that activation of the cGMP-system induced an increase of dye loading during high-frequency stimulation (Fig. $2 B, D$ ) which could be associated with the significant increase in fast endocytosis of SVs. This is the likely explanation of the observation that $60 \%$ of fused vesicles were retrieved through endocytosis during highfrequency stimulation as opposed to $30 \%$ in control conditions (Fig. 2D).

The combination of our electrophysiological data and fluorescence measurements revealed two-fold acceleration of SVs cycling after activation of the cGMP-pathway (Fig. $4 B, C)$. The total capture of dye after cGMP application was lower than under control conditions, because SVs could lose the dye during application of FM1-43. The summed exocytosis during 3 min of stimulation was equal to that under control conditions (Fig. 1D).

The increase of vesicle recycling after treatment with cGMP analogs may underlie the time course of changes of the EPCs amplitude during high-frequency stimulation. The reduced depression of release during the first $30 \mathrm{~s}$ of stimulation could be linked to the increased recruitment of SVs from mobilization pool in transmitter release because of more rapid delivery of these vesicles to RRP. However, an increased recycling rate of SVs cannot fully support transmitter release during prolonged highfrequency activity because of the eventual depletion of the recycling pool (including the RRP) (Fig. $1 B, C$ ).

\section{The decrease of cGMP production suppresses fast endocytosis} of SV by short pathway during high-frequency stimulation

The inhibition of soluble guanylate cyclase led to effects on endocytosis and vesicle recycling during high-frequency stimulation that were opposite to those associated with activation of the cGMP system. The decrease of dye loading during stimulation and the increased duration of the period of dye capture after stimulation (Fig. 2B) suggests a depression of fast endocytosis. The complete coincidence of the curves of dye loss and summed EPCs amplitude (Fig. 4D) demonstrates the absence of SVs reuse by the short pathway in transmitter release.

Inhibition of guanylate cyclase markedly slowed the destaining of FM1-43 during prolonged high-frequency activity, as the first fast phase of nerve terminal destaining was disappeared. The fist phase of destaining is usually explained by the exit of dye from vesicles of the recycling pool, while the second slow phase depends on the exocytosis dye containing vesicles from reserve pool (Richards et al., 2003). In our case, dye destaining after inhibition of guanylate cyclase may reflect the use of vesicles of the reserve pool in exocytosis and transmitter release. Pronounced and rapidly developed depression of EPCs after ODQ treatment also indicates an impairment of SVs traffic from the mobilization pool to the RRP (Fig. $1 B, C$ ). As a result, the summed EPCs amplitude during 3 min of high-frequency stimulation was $40 \%$ lower than in control experiments (Fig. 1D).

These results suggest the existence of a tonic level of guanylate cyclase activity in motor nerve terminals, maintaining a background concentration of cGMP, which supports the endocytosis machinery and recycling of SVs during high-frequency activity. cGMP production, in turn, depends on the intracellular concentration of $\mathrm{Ca}^{2+}$, ATP and retrograde messengers, including gases (such as NO), secreted from the neighboring cells (Lev-Ram et al., 1997; Sitdikova et al., 2007).

It is well-known that high-frequency stimulation can lead to sequential depletion of recycling and reserve vesicle pools, although the underlying molecular mechanisms of these processes remain obscure (Rizzoli and Betz, 2005). Based on our findings, we propose that SVs of the mobilization and reserve pools could be released simultaneously. A reduced contribution of the recycling pool in transmitter release after ODQ treatment, while the reserve pool is still active, supports the possibility of two independent vesicle cycles in the frog motor nerve terminal.

\section{Possible molecular targets of cGMP system}

The nature of the effects of guanylate cyclase and PKG inhibition in our study indicates that PKG mediates cGMP action in motor nerve terminals. In our experiments, a low concentration of the PKG inhibitor Rp-8-Br-PET-cGMP(0.5 $\mu \mathrm{M})$ was used which affects mainly the activity of PKG1 (Schwede et al., 2000). Therefore, PKG1 is likely to be the main target of cGMP in frog motor nerve terminals.

What are the potential molecular targets of PKG? Recent studies suggest that phosphoinositide metabolism plays an important role in synaptic membrane trafficking (Wenk and De Camilli, 2004). PKG1 was suggested to increase the membrane pool of PtdIns4,5P2 (Micheva et al., 2003), both through inhibition of its degradation by phospholipase $\mathrm{C}$ and the phosphorylation of the small GTPases that control the activity of PIP kinases (Clementi et al., 1995; Rohrbough and Broadie, 2005).

The effects of cGMP on SVs recycling may be linked to the changes of vesicle trafficking in presynaptic terminal after endocytosis. It has been shown that PKG1 affects hsp27 and Ena/VASP proteins, several small GTPases, regulating vector actin polymerization (Butt et al., 2001; Gomez and Robles, 2004). In addition, PKG can inhibit myosin-based motors influencing the processes of phosphorylation-dephosphorylation of myosin light chains (Wang et al., 2005). Another possibility relates to the phosphorylation of G-septin by PKG. Septins are a family of GTPase enzymes, primarily concentrated in the nerve terminal (Xue et al., 2004), which are able to assemble into polymers and to act as diffusion barriers between different subcellular compartments. Septins might therefore tether SVs, including the vesicles of mobilization pool, to the region near the active zones and thus prevent their trafficking to the reserve pool. In addition, septins could provide the initial interaction between the membrane of SV and syntaxin in the membrane of active zone (Spiliotis and Nelson, 2006).

The data obtained in this study show that the cGMP-PKG1 system directly participates in the vesicle cycle through a regulation of SVs endocytosis and vesicle trafficking in the presynaptic terminals. Therefore, molecular manipulations of the SVs recycling machinery not only help us to uncover the mechanisms of vesicle trafficking but also provide an extremely valuable setting in which to study the kinetics and physiological significance of SVs reuse during synaptic activity.

\section{References}

Becherer U, Rettig J (2006) Vesicle pools, docking, priming and release. Cell Tissue Res 326:393-407.

Becherer U, Guatimosim C, Betz WJ (2001) Effects of staurosporine on exocytosis and endocytosis at frog motor nerve terminals. J Neurosci 21:782-787.

Betz WJ, Bewick GS (1993) Optical monitoring of transmitter release and synaptic vesicle recycling at the frog neuromuscular junction. J Physiol 460:287-309. 
Betz WJ, Henkel AW (1994) Okadaic acid disrupts clusters of synaptic vesicle in frog motor nerve terminals. J Cell Biol 142:843-854.

Butt E, Immler D, Meyer HE, Kotlyarov A, Laass K, Gaestel M (2001) Heat shock protein 27 is a substrate of cGMP-dependent protein kinase in intact human platelets: phosphorylation-induced actin polymerization caused by Hsp27 mutants. J Biol Chem 276:7108-7113.

Clementi E, Sciorati C, Riccio M, Miloso M, Meldolesi J, Nistico G (1995) Nitric oxide action on growth factor-elicited signals. Phosphoinositide hydrolysis and $\left[\mathrm{Ca}^{2+}\right]_{\mathrm{i}}$ responses are negatively modulated via a cGMPdependent protein kinase I pathway. J Biol Chem 270:22277-22282.

Cousin MA, Robinson PJ (2001) The dephosphins: dephosphorylation by calciuneurin triggers synaptic vesicle endocytosis. Trends Neurosci 24:659-665.

Couteaux R, Pécot-Dechavassine M (1970) Synaptic vesicles and pouches at the level of "active zone" of the neuromuscular junction. C R Acad Sci Hebd Seances Acad Sci D 271:2346-2349.

Dai Z, Peng HB (1996) Dynamics of synaptic vesicles in cultured spinal cord neurons in relationship to synaptogenesis. Mol Cell Neurosci 7:443-452.

Elmqvist D, Hofmann WW, Kugelberg J, Quastel DM (1964) An electrophysiological investigation of neuromuscular transmission in myasthenia gravis. J Physiol 174:417-434.

Feil R, Hartmann J, Luo C, Wolfsgruber W, Schilling K, Feil S, Barski JJ, Meyer M, Konnerth A, De Zeeuw CI, Hofmann F (2003) Impairment of LTP and cerebellar learning by Purkinje cell-specific ablation of cGMPdependent protein kinase 1. J Cell Biol 163:295-302.

Glavinović MI (1979) Voltage clamping of unparalysed cut rat diaphragm for study of transmitter release. J Physiol 290:467-480.

Gomez MT, Robles E (2004) The great escape: phosphorylation of Ena/ VASP by PKA promotes filopodial formation. Neuron 42:1-3.

Kavalali ET (2006) Synaptic vesicle reuse and its implications. Neuroscientist 12:57-66.

Kleppisch T, Wolfsgruber W, Feil S, Allmann R, Wotjak CT, Goebbels S, Nave KA, Hofmann F, Feil R (2003) Hippocampal cGMP-dependent protein kinase I supports an age-and protein synthesis-dependent component of long-term potentiation but is not essential for spatial reference and contextual memory. J Neurosci 23:6005-6012.

Kraszewski K, Daniell L, Mundigl O, DeCamilli P (1996) Mobility of synaptic vesicles in nerve endings monitored by recovery from photobleaching of synaptic vesicle-associated fluorescence. J Neurosci 16:5906-5913.

Kuromi H, Kidokoro Y (2005) Exocytosis and endocytosis of synaptic vesicle and functional roles of vesicle pools: lessons from the Drosophila neuromuscular junction. Neuroscientist 11:138-147.

Lev-Ram V, Jiang T, Wood J, Lawrence DS, Tsien RY (1997) Synergies and coincidence requirements between NO, cGMP, and $\mathrm{Ca}^{2+}$ in the induction of cerebellar long-term depression. Neuron 18:1025-1038.

Micheva KD, Buchanan J, Holz RW, Smith SJ (2003) Retrograde regulation of synaptic vesicle endocytosis and recycling. Nat Neurosci 6:925-932.

Reid B, Martinov VN, Njå A, Lømo T, Bewick GS (2003) Activity-depend plasticity of transmitter release from nerve terminals in rat fast and slow muscles. J Neurosci 23:9340-9348.

Richards DA, Guatimosim C, Betz WJ (2000) Two endocytic recycling routes selectively fill two vesicle pools in frog motor nerve terminals. Neuron 27:551-559.

Richards DA, Guatimosim C, Rizzoli SO, Betz WJ (2003) Synaptic vesicle pools at the frog neuromuscular junction. Neuron 39:529-541.

Rizzoli SO, Betz WJ (2005) Synaptic vesicle pools. Nat Rev Neurosci 6:57-69.

Rohrbough J, Broadie K (2005) Lipid regulation of the synaptic vesicle cycle. Nat Rev Neurosci 6:139-150.

Schwede F, Maronde E, Genieser H, Jastorff B (2000) Cyclic nucleotide analogs as biochemical tools and prospective drugs. Pharmacol Ther 87:199-226.

Sitdikova GF, Islamov RR, Mukhamedyarov MA, Permykova VV, Zefirov AL, Palotás A (2007) Modulation of neurotransmitter release by carbon monoxide at the frog neuro-muscular junction. Curr Drug Metab 8:177-184.

Snyder DA, Kelly ML, Woodbury DJ (2006) SNARE complex regulation by phosphorilation. Cell Biochem Biophys 45:111-123.

Spiliotis ET, Nelson WJ (2006) Here come the septins: novel polymers that coordinate intracellular functions and organization. J Cell Sci 119:4-10.

Stanton PK, Winterer J, Bailey CP, Kyrozis A, Raginov I, Laube G, Veh RW, Nguyen CQ, Müller W (2003) Long-term depression of presynaptic release from the readily releasable vesicle pool induced by NDMA-receptordependent retrograde nitric oxide. J Neurosci 23:5936-5944.

Sudhof TC (2004) The synaptic vesicle cycle. Annu Rev Neurosci 27:509-547.

Tabares L, Ruiz R, Linares-Clemente P, Gaffield MA, Toledo GA, FernandezChacón R, Betz WJ (2007) Monitoring synaptic function at the neuromuscular junction of a mouse expressing synaptopHluorin. J Neurosci 27:5422-5430.

Thomas S, Robitaille R (2001) Differential frequency-depend regulation of transmitter release by endogenous nitric oxide at the amphibian neuromuscular synapse. J Neurosci 21:1087-1095.

Wang HG, Lu FM, Jin I, Udo H, Kandel ER, de Vente J, Walter U, Lohmann SM, Hawkins RD, Antonova I (2005) Presynaptic and postsynaptic roles of NO, cGC, and RhoA in long-lasting potentiation and aggregation of synaptic proteins. Neuron 45:389-403.

Wenk MR, De Camilli P (2004) Protein-lipid interaction and phosphoinositide metabolism in membrane traffic: insights from vesicle recycling in nerve terminals. Proc Natl Acad Sci U S A 101:8262-8269.

Xue J, Tsang CW, Gai WP, Malladi CS, Trimble WS, Rostas JA, Robinson PJ (2004) Septin 3 (G-septin) is a developmentally regulated phosphoprotein enriched in presynaptic nerve terminals. J Neurochem 91:579-590.

Yakovlev A, Sitdikova G, Zefirov A (2004) Intracellular presynaptic mechanisms of nitric oxide (2) action in frog neuromuscular junction (in Russian). Neirokhimiya 21:325-331.

Zefirov AL, Grigoriev PN, Petrov AM, Minlebaev MG, Sitdikova GF (2003) Fluorescent investigation of the living motor nerve ending of the frog by the endocytotic marker FM 1-43 (in Russian). Tsitologiia 45:1163-1171.

Zefirov AL, Abdrakhmanov MM, Mukhamedyarov MA, Grigoryev PN (2006) The role of extracellular calcium in exo- and endocytosis of synaptic vesicles at the frog motor nerve terminals. Neurosci 143:905-910.

Zefirov AL, Zakharov AV, Mukhamedzjanov RD, Petrov AM, Sitdikova GF (2008) Vesicle cycle in mouse diaphragm motor nerve terminals. Ross Fiziol Zh Im I M Sechenova (Russian) 94:129-141. 